\title{
A class of modules over a locally finite group III
}

\section{B. Hartley}

Let $G$ be a locally finite group, $k$ a field of characteristic $p \geq 0$ and $V$ a right $k G$-module. We say that $V$ is an $\underline{\underline{M}} c^{- \text {module over }} k G$, if each $p^{\prime}$-subgroup $H$ of $G$ contains a finite subgroup $F$ with the same fixed points as $H$ in $V$. (By convention, $0^{\prime}$ is taken as the set of all primes.) Such modules arise as elementary abelian sections of $\underline{\underline{U}}$-groups, a class of locally finite groups similar in many ways to the class of finite soluble groups.

The main theorem is that if $V$ is an $\underline{\underline{M}}_{c}$-module over $k G$ with trivial Frattini submodule, and $G$ is almost abelian, then every composition factor of $V$ is complemented. This is a crucial ingredient in Tomkinson's theory of prefrattini subgroups in a certain subclass of $\underline{\underline{U}}$. An example is given to show that the theorem breaks down for metabelian $G$. This leads to an example of a $\underline{\underline{U}}$-group in which there are no analogues of prefrattini subgroups - the first situation where one of the standard conjugacy classes of subgroups of finite soluble groups has no decent analogue in the whole class $\underline{\underline{U}}$.

\section{Introduction}

Let $G$ be a locally finite group, let $k$ be a field of characteristic $p \geq 0$, and let $V$ be a (right) $k G$-module. In the terminology of [5] and [6], $V$ is an $\underline{M}_{c}$-module over $k G$, if each

Received 6 November 1975. The author wishes to record his thanks to the University of Wisconsin in Madison for its hospitality and support while this paper was being written. 
$p^{\prime}$-subgroup $H$ of $G$ contains a finite subgroup $F$ such that $C_{V}(F)=C_{V}(H)$. Here, $O^{\prime}$ is interpreted as the set of all primes, and $C_{V}(H)$ denotes the set of all elements of $V$ fixed by every element of $H$. If $G$ itself is a $p^{\prime}$-group, it follows easily that the set $\left\{C_{V}(H): H \leq G\right\}$ satisfies the minimal condition under inclusion, and it is for this reason that the $\underline{M}_{C}$ notation is used.

In [5], we were interested in conditions ensuring the complete reducibility of $\underline{M}_{c}$-modules. Before passing to the main topic of this paper, we mention an improvement of the results of [5] which can be obtained on the basis of recent results of Richardson [10]. Let $H$ be a locally finite group, and let $k$ be a field with algebraic closure $\bar{k}$ and prime field $k_{0}$. Let $k_{0}(H)$ be the subfield of $\bar{k}$ generated by all roots of the polynomials $x^{n}-1$, as $n$ runs over the orders of the elements of $H$. If $k_{0}(H) \cap k$ has finite dimension over $k_{0}$, we shall say that $k$ is far enough from $H$. In particular, this happens if $H$ is a finite group, or if $k$ is a finite algebraic extension of $k_{0}$. By the arguments of [5], incorporating [10], 3.4 instead of [5], Lemma 2.3, we obtain

THEOREM A. Let $G$ be a Ternikov group, let $k$ be a field of characteristic $p \geq 0$, and let $V$ be an $\underline{M}_{c}$-module over $k G$. Suppose that $k$ is far enough from $G$, and that either $G$ is a $p^{\prime}$-group or the Frattini submodule $\Phi(V)=0$. Then $V$ is completely reducible.

By a Černikov group, we mean as usual a finite extension of a direct product of finitely many quasi-cyclic groups. Thus, Maschke's Theorem is a special case of the above.

If $k$ is a finite field of characteristic $p$ and $G$ is an abelian $p^{\prime}$-group which is not Černikov, then an $\underline{M}_{c}$-module $V$ over $k G$ may well fail to be completely reducible (see [5], Example 4.1). But by a result of Kovács and Newman [8], which does not depend on the $M_{c}$-condition, the minimal submodules of $V$, if any, will be complemented. Since the same holds for factor modules of $V$, every composition factor $A / B$ of $V$ will 
also be complemented, in the sense that $V=A+C$ and $A \cap C=B$ for some submodule $C$ of $V$. In fact, we only need to assume that $G$ is almost abelian (that is, has an abelian subgroup of finite index) here, by more recent work of Farkas and Snider [1]; see also [7].

The aim of this paper is to consider the complementation of the composition factors when $\Phi(V)=0$ but $G$ is not assumed to be a p'-group.

THEOREM B. Let $G$ be a periodic almost abelian group, let $k$ be any field, and let $V$ be an $\underline{M}_{c}$-module over $k G$ with $\Phi(V)=0$. Then every composition factor of $V$ is complemented.

Here, the condition that $V$ be an $\stackrel{M}{\Rightarrow}$-module is not superfluous. For let $A$ be any infinite abelian group containing no elements of order 2 , and let $G$ be the semidirect product of $A$ by an automorphism of order 2 which inverts the elements of $A$. If $k$ is the field of two elements, the group ring $k G$ is semisimple ([9], 2l.1) and so, viewed as a right module over itself, has zero Frattini submodule. But $k G$ has the group ring of a cyclic group of order 2 over $k$ as homomorphic image, and this group ring contains a minimal right ideal which is not complemented.

The study of $\underline{M}_{C}$-modules has developed from the investigation of sylow subgroups of locally finite groups, and particularly from the study of a class $\underline{\underline{U}}$ of locally finite-soluble groups whose properties are in many ways analogous to those of the class of finite soluble groups (see [2]). Theorem $B$ has already been applied to the class $\underline{\underline{U}}$ by Tomkinson [11] in developing the theory of prefrattini subgroups of $\underline{\underline{U}}$-groups.

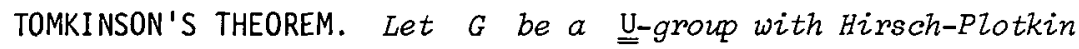
radical $\rho(G)$, and suppose that $G / \rho(G)$ is almost abelian. Then each prefrattini subgroup of $G$ avoids the complemented chief factors of $G$ and covers the non-complemented chief factors of $G$.

The second aim of this paper is to show that Tomkinson's Theorem breaks down in the class of $\underline{\underline{U}}$-groups as a whole. Now the prefrattini subgroups are not characterized by the covering and avoiding properties described above, even in finite soluble groups [3], but in our discussion we shall not need to know precisely what the prefrattini subgroups are. 
We prove

THEOREM C. Let $r$ be any prime. Then there exists a group $G \in \underline{\underline{U}}$ and an $\underline{\underline{M}}_{c}$-moduze $W$ over $\mathrm{Z}_{p} G$ such that $\Phi(W)=0$, but $W$ has an indecomposable quotient module of composition length two.

Let $H$ be the semidirect product $H=W G, W$ being written multiplicatively. Then $H \in \underline{\underline{U}}$ ([4], Lemma 7.1). Let $X$ be any maximal submodule of $W$. In $H, W / X$ is a chief factor complemented by $G X / X$, and so, if $D$ is any subgroup of $H$ which avoids all the complemented chief factors, $D \cap W \leq X$. Since $\Phi(W)=0, D \cap W=1$. However, there exist submodules $T<S<W$ such that $S / T$ is irreducible and not complemented in $W / T$. In $H, S / T$ is a non-complemented chief factor. Thus, $H$ has a non-complemented chief factor which $D$ avoids, and Theorem C has the

COROLLARY. There exists a U-group $H$ such that every subgroup of $H$ which avoids all the complemented chief factors, avoids also a noncomplemented chief factor.

The prefrattini subgroups seem to be the first example of one of the standard conjugacy classes of subgroups from the theory of finite soluble groups which have no well behaved analogue in the class $\underline{U}$ as a whole. However, Tomkinson's Theorem shows that even they behave well in some of the more familiar subclasses of $\underline{\underline{U}}$, such as the class of images of periodic soluble linear groups, or the class of periodic, locally soluble, almost locally nilpotent groups.

\section{Local complete reducibility}

Since it is difficult to see how the vanishing of the Frattini submodule of a module influences its quotient modules, we shall prove Theorem $\mathrm{B}$ by showing that the modules in which we are interested have another property, which clearly does pass to quotient modules, and using that to obtain the complementation of the composition factors. This is the property of strong local complete reducibility.

DEFINITION. Let $G$ be a locally finite group, $k$ a field, and $V$ a $k G$-module. We say that $V$ is strongly locally completely reducible if there exists a finite subgroup $F_{1}$ of $G$ such that the restricted module 
$V_{F}$ is completely reducible for all finite subgroups $F \geq F_{1}$ of $G$.

This, on the face of it, is stronger than demanding that $G$ have a local system $X$ of finite subgroups $X$ such that $V_{X}$ is completely reducible for all $X \in X$ - the natural concept of local complete reducibility.

We now study this notion, beginning with the case when $k$ contains all roots of 1 . Throughout the rest of this section, $G$ denotes a locally finite group containing an abelian normal subgroup $H$ of finite index, $k$ denotes a field and $V$ a $k G$-module.

LEMMA 2.1. Let $L$ be any subgroup of $H$. There exists a finite set $X$ of elements of $G$ such that, if $F$ is any subgroup of $G$ containing $X$, then

$$
F \cap C_{G}(H / L)=C_{F}((F \cap H) /(F \cap L)) .
$$

Here, if $A$ and $B$ are subgroups of a group $Y$ with $B \triangleleft A$, we write

$$
C_{Y}(A / B)=\left\{y \in Y:\left[a, y^{ \pm 1}\right] \in B \text { for all } a \in A\right\} \text {. }
$$

Thus an element $y \in Y$ belongs to $C_{Y}(A / B)$ if and only if $y$ normalizes both $A$ and $B$ and operates trivially by conjugation on $A / B$. Therefore $C_{Y}(A / B)$ is a subgroup of $Y$.

Proof. Let $C=C_{G}(H / L) \geq H$, and let $1=s_{1}, s_{2}, \ldots, s_{n}$ be a left transversal to $C$ in $G$. For each $i=2, \ldots, n$, there exists an element $h_{i} \in H$ such that $\left[h_{i}, s_{i}\right] \neq L$ (recall that $G$ is periodic). Let $X=\left\{s_{2}, \ldots, s_{n}, h_{2}, \ldots, h_{n}\right\}$ and let $F$ be a subgroup of $G$ containing $X$. Clearly $F=\bigcup_{i=1}^{n} s_{i}(F \cap C)$, and $F \cap C$ centralizes $(F \cap H) /(F \cap L)$, whereas $s_{2}, \ldots, s_{n}$ do not. Therefore $F \cap C=C_{F}((F \cap H) /(F \cap L))$.

LEMMA 2.2. Suppose that $V$ is an irreducible kG-module, and $k$ contains all roots of $I$. Then the restricted module $V_{H}$ is completely 
reducible. Let $Y$ be an irreducible submodule of $V_{H}$, let $L=C_{H}(Y)$, and suppose $F$ is a subgroup of $G$ such that $G=H F$ and $F$ has the property (*) with respect to $L$. Then $V_{F}$ is irreducible.

Proof. Since $H$ is a normal subgroup of finite index of $G$, Clifford's Theorem ([6], Lemma 2.II) shows that $V_{H}$ is completely reducible, and is the direct sum of finitely many irreducible $k H$-submodules. Since $H$ is periodic abelian and $k$ contains all roots of 1 , each of these is one-dimensional, so that in fact $\operatorname{dim}_{k} V<\infty$. It follows easily that there exist finite subgroups $E$ of $G$ such that $V_{E}$ is irreducible, since the image of $k G$ in end $V$ is finite-dimensional and so can be spanned by finitely many elements of $G$. However we require more precise information in the sequel.

Let

$$
V_{H}=y_{I} \oplus \ldots \oplus Y_{s}
$$

be the decomposition of $V_{H}$ into its homogeneous components, with $Y \leq Y_{1}$. Since $G=H F$, there exist elements $1=x_{1}, x_{2}, \ldots, x_{s} \in F$ such that $y_{i}=y_{1} x_{i}$. If $i \geq 2$, then $Y x_{i}$ is not $k H$-isomorphic to $Y$, and so $x_{i} \vDash C=C_{G}(H / L)$, and since $F$ has the property (*), $x_{i} \neq C_{F}((F \cap H) /(F \cap L))$. Therefore $Y$ and $Y x_{i}$ are non-isomorphic $k(F \cap H)$-modules (see [6], Lemma 5.1), and (1) is the decomposition of $V_{F \cap H}$ into its homogeneous components. Now let $W$ be any non-zero $k F$-submodule of $V$. Restricting $W$ to $F \cap H$, we see that $z=W \cap Y_{i} \neq 0$ for some $i$. Since $H$ operates by scalar multiplication on $Y_{i}$, every subspace of $Y_{i}$, and $Z$ in particular, is $H$-invariant. Therefore, since $G=H F, \sum_{f \in F} Z f$ is a non-zero $k G$-submodule of $V$. Since it lies in $W$, while $V$ is irreducible, we obtain $W=V$.

Some of our subsequent arguments will involve carrying out field extensions, and the following fact will be crucial.

LEMMA 2.3. Let $A$ be any k-algebra, let $W$ be any A-module, and 
let $K$ be a separable algebraic extension of $k$. If $\Phi(W)=0$ and $W \otimes_{k} K$ is regarded as an $A \otimes_{k} K$-module in the usual way, then $\Phi\left(W \otimes_{k} K\right)=0$.

Proof. If $W_{1}$ is any maximal submodule of $W$, then

$$
\left(W \otimes_{k} K\right) /\left(W_{1} \otimes_{k} K\right) \cong\left(W / W_{1}\right) \otimes_{k} K
$$

and $n\left(W_{1} \otimes_{k} K\right)=0$ as $W_{1}$ runs over all maximal submodules of $W$, since $\Phi(W)=0$. Therefore, we may assume that $W$ is irreducible. Let $v$ be any non-zero element of $W \otimes_{k} K$. There exists a finite separable algebraic extension $k_{I}$ of $k$, contained in $K$, such that $v \in W \otimes_{k} k_{1}$. From [9], Chapter III, see in particular Lemma 15.4, we see that $W \otimes_{k} k_{1}$ is completely reducible as $A \otimes_{k} k_{1}$-module, and so there exists a maximal submodule $M$ of $W \otimes_{k} k_{1}$ not containing $v$. Let $\bar{M}=M \otimes_{k_{1}} K$, which is a proper $A \otimes_{k} K$-submodule of $W \otimes_{k} K$, and let $N$ be a maximal submodule of $W \otimes_{k} K$ containing $\bar{M}$. Such a maximal submodule exists since $W \otimes_{k} K$ is a cyclic $A Q_{k} K$-module, being generated by any non-zero element of $W$. For this reason also, $N \cap\left(W \otimes_{k} k_{1}\right)=M$. Therefore $v k N$, and it follows that $\Phi\left(W \otimes_{k} K\right)=0$.

The next result is not strictly relevant to the main theorems of the paper, but nevertheless seems of some interest.

PROPOSITION 2.4. If $V$ is irreducible then it is strongly localzy completely reducible.

Proof. Let $K$ be a field containing and generated by $k$ and all roots of 1 . Then $k$ is a separable algebraic extension of $k$, and so by Lemma 2.3, if $\bar{V}$ denotes the $K G$-module $V \Theta_{k} K$, we have $\Phi(\bar{V})=0$.

Now $V_{H}$ is completely reducible by Clifford's Theorem, and we can write

$$
V_{H}=U_{1} \oplus \cdots \oplus U_{s}
$$


where the $U_{i}$ are irreducible $k H$-modules. If $\left\{\omega_{\lambda}\right\}$ is a basis of $K$ over $k$, then $\bar{V}=\underset{\lambda}{\oplus} \underset{\lambda}{\oplus} \omega_{\lambda}$, and we find that, as a $k H$-module, $\bar{V}$ is completely reducible into a direct sum of irreducible submodules each isomorphic to some $U_{i}$. The same holds for any $k H$-factor $A / B$ of $\bar{V}$, and in particular for any $K H$-composition factor $X$ of $\bar{V}$. Now if $U$ is any irreducible $k H$-submodule of $X$, then $\sum_{0 \neq \alpha \in K} U \alpha$ is a $K H$-submodule, and is a direct sum of copies of $U$ as $k H$-module. Therefore every $K H$-composition factor $X$ of $\bar{V}$ is a direct sum of copies of some $U_{i}$, and if $L_{i}=C_{H}\left(U_{i}\right)$, then $L_{i}=C_{H}(X)$.

Let $T$ be any maximal KG-submodule of $\bar{V}$. Then as in Lemma 2.2, $\vec{V} / T$ contains an irreducible $K H$-submodule $Y / T$, and, by the above, $C_{H}(Y / T)$ is one of $L_{1}, \ldots, L_{s}$. By Lemma 2.1 , there exists a finite subgroup $F_{1}$ of $G$ such that every finite subgroup $F \geq F_{1}$ of $G$ has the property (*) with respect to each of $L_{1}, \ldots, L_{s}$, and such that $H F_{1}=G$. By Lemma 2.2, $\bar{V} / T$ is an irreducible $K F$-module for all such $F$. Therefore $\Phi\left(V_{F}\right)=0$.

It follows that the Jacobson radical of $K F$ annihilates $\bar{V}$, and hence that the Jacobson radical of $k F$, which, being nilpotent, lies in that of $K F$, annihilates $V$. Therefore $V_{F}$ is completely reducible, and the result is established.

Passman has pointed out that Proposition 2.4 may also be proved rather easily using the fact that $V$ is finite-dimensional over its endomorphism ring ([9], Chapter II, 5.1 and 6.4 , or [7]).

The next result provides the key to the proof of Theorem B.

LEMMA 2.5. If $V$ is an $\underline{M}_{c}$-module over $k G$ and $\Phi(V)=0$, then $V$ is strongly locally completely reducible.

Proof. Let $K$ be a field containing and generated by $k$ and all roots of 1 , and let $\bar{V}$ be the $K G$-module $V \otimes_{k} K$. By Lemma 2.3, $\Phi(\bar{V})=0$. Also, $\bar{V}$ is an $\underline{M}_{C}$-module over $K G$. For let $p \geq 0$ be the 
characteristic of $k$, and let $H$ be any $p^{\prime}$-subgroup of $G$. Then as $V$ is an $\underline{\underline{M}}_{C}$-module, $C_{V}(H)=C_{V}(E)$ for some finite subgroup $E$ of $H$. But as $k G$-module, $\bar{V}$ is a direct sum of copies of $V$. Therefore $C_{\bar{V}}(H)=C_{\bar{V}}(E)$, and $\bar{V}$ is an $\underline{M}_{C}$-module over $K G$. Finally, if $F$ is a finite subgroup of $G$ such that $\vec{V}_{F}$ is completely reducible, then $V_{F}$ is completely reducible, by the argument at the end of the proof of Proposition 2.4. Therefore we may assume that $k$ contains ald roots of 1 .

We may also assume that $V$ is faithful for $G$. Since $o_{q}(G)$ operates trivially on every irreducible module for $G$ over a field of prime characteristic $q$, and $\Phi(V)=0$, it follows that $o_{p}(G)=1$ if the characteristic $p$ of $k$ is a prime. In particular, $H$ is a $p^{\prime}$-group. Also, $H$ has finite (Mal'cev special) rank, and in particular its Sylow subgroup $H_{q}$ satisfies min for each prime $q$. This follows from [6], Lemma 3.4 , or [4].

Let $\left\{M_{\lambda}: \lambda \in \Lambda\right\}$ be the set of all maximal submodules of $V$. Then as in Lemma 2.2, $V / M_{\lambda}=V_{\lambda}$ is an irreducible $k G$-module of finite dimension over $k$. Let $y_{\lambda}$ be an irreducible $k H$-submodule of $V_{\lambda}$ and let $N_{\lambda}=C_{H}\left(y_{\lambda}\right)$. In order to apply the methods used in proving Proposition 2.4, we need to study the family $\left\{N_{\lambda}\right\}$ of subgroups of $H$.

Let $Y$ be a finite subgroup of $G$ such that $G=H Y$ and let $\pi$ be the set of prime divisors of $|Y|$. Then $\pi$ is finite. Let $y \in Y$. Since $V$ is an $\underline{\underline{M}}_{C}$-module and $H$ is a $p^{\prime}$-group, $C_{V}\left(\left[H_{\pi}, y\right]\right)=C_{V}\left(E_{y}\right)$ for some finite subgroup $E_{y}$ of $\left[H_{\pi}, y\right]$, which may be taken to be $\langle y\rangle$-invariant. Now we trivially have

$$
C_{V_{\lambda}}\left(E_{y}\right) \geq C_{V_{\lambda}}\left(\left[H_{\pi^{\prime}}, y\right]\right) \text {. }
$$

But since $E_{y}$ is a finite $p^{\prime}$-group, a simple local argument allows. us to deduce from the finite dimensional case that $C_{V_{\lambda}}\left(E_{y}\right)$ is the natural image in $V_{\lambda}$ of $C_{V}\left(E_{y}\right)$. Since this is centralized by $\left[H_{\pi}, y\right]$, the 
inclusion in (3) can be replaced by equality. In particular,

$$
N_{\lambda} \geq E_{y} \Leftrightarrow N_{\lambda} \geq\left[H_{\pi}, y\right] \text {. }
$$

By Fitting's Lemma, $y$ fixes no non-trivial element of $\left[H_{\pi^{\prime}}, y\right]$, and hence $E_{y}=\left[E_{y}, y\right]$. Since $H$ satisfies min-q for all primes $q$, $E_{y}$ lies in a finite characteristic $\pi$ '-subgroup $D_{y}$ of $H$. Let $D=\prod_{y \in Y} D_{y}$. Then $D$ is a finite characteristic subgroup of $H$, and we claim that

$$
C_{G}\left(H_{\pi}, /\left(H_{\pi}, W_{\lambda}\right)\right)=C_{G}\left(D /\left(D \cap N_{\lambda}\right)\right)
$$

for all $\lambda$. In fact, suppose that an element $g \in G$ centralizes $D /\left(D N N_{\lambda}\right)$. Write $g=h y$ with $y \in Y$. Then $y$ centralizes $D /\left(D N N_{\lambda}\right)$. Therefore $E_{y}=\left[E_{y}, y\right] \leq D \cap N_{\lambda}$. By (4), $\left[H_{\pi}^{\prime}, y\right] \leq N_{\lambda}$, and so $g$ centralizes $H_{\pi}, /\left(H_{\pi}, W_{\lambda}\right)$. Thus (5) holds.

Now consider the subgroups $H_{\pi} \cap N_{\lambda}(\lambda \in \Lambda)$. If infinitely many of these are distinct, then it is not hard to see ([5], Lemma 3.2) that there exists a subgroup $B$ of $H_{\pi}$ such that $B=\bigcup_{i=0}^{\infty} B_{i}$ is the union of a tower $B_{0} \leq B_{1} \leq \ldots$ of subgroups, each of which lies in infinitely many distinct subgroups $H_{\pi} \cap N_{\lambda}$, while $B$ itself lies in only finitely many. Let $A$ be a finite subgroup of $B$ such that $C_{V}(A)=C_{V}(B)$. As above, we find that $N_{\lambda} \geq A$ if and only if $N_{\lambda} \geq B$. Since $A$ lies in one of the subgroups $B_{i}$, we have a contradiction. Thus, only finitely many of the subgroups $H_{\pi} \cap N_{\lambda}$ are distinct, and by Lemma 2.1, there exists a finite subgroup $F_{2}$ of $H_{\pi} Y$ such that every subgroup of $H_{\pi} Y$ containing $F_{2}$ has the property (*), with $H_{\pi}$ playing the role of $H$ and each of $H_{\pi} \cap N_{\lambda}$ playing the role of $L$. Here $F_{2}$ is independent of $\lambda$, and we may suppose $F_{2} \geq Y$. Let $F_{1}=D F_{2}$ and let $F$ be a finite subgroup of $G$ containing $F_{1}$. We claim that $F$ has the property (*) with respect to 
each of the $N_{\lambda}$ as $L$. For if $f \in F$ centralizes $(F \cap H) /\left(F \cap N_{\lambda}\right)$, write $f=h y$ with $h \in H, y \in Y$. Then $y$ centralizes $(F \cap H) /\left(F \cap W_{\lambda}\right)$, and so centralizes $D /\left(D \cap N_{\lambda}\right)$. By (5), y centralizes $H_{\pi^{\prime}} /\left(H_{\pi^{\prime}}, N_{\lambda}\right)$. The property of $F_{2}$ ensures that $y$ centralizes $H_{\pi} /\left(H_{\pi} \cap N\right)$. Hence $y$ centralizes $H /\left(H \cap N_{\lambda}\right)$ and so does $f$, as claimed.

It now follows from Lemma 2.2 that each $V_{\lambda}=V / M_{\lambda}$ remains irreducible when restricted to any finite subgroup $F \geq F_{1}$ of $G$. Therefore $\Phi\left(V_{F}\right)=0$. But then all submodules of $V_{F}$, and in particular those finite-dimensional over $k$, have trivial Frattini submodule. Since $V_{F}$ is a sum of finite-dimensional $k F$-modules, and these are completely reducible since their Frattini submodules vanish, $V_{F}$ is complete reducible. This establishes Lemma 2.5.

Theorem $B$ follows from the somewhat stronger version below.

THEOREM $B^{\prime}$. Let $G$ be a periodic almost abelian group, $k$ a field and $V$ an ${ }_{c}$-module over $k G$. The following three conditions are equivalent:

(i) $\Phi(V)=0$;

(ii) $V$ is strongly locally completely reducible;

(iii) every composition factor of $V$ is complemented.

It will be seen that some implications between these conditions can be obtained without the $\underline{\underline{M}}_{C}$-condition.

Proof. (i) $\Rightarrow$ (ii) This is given by Lemma 2.5 .

(ii) $\Rightarrow$ (iii) We shall prove this implication without using the $\underline{\underline{M}}_{c}$-condition. Since the property of being strongly locally completely reducible is inherited by quotient modules of $V$, it suffices to show that every minimal submodule $U$ of $V$ is complemented. Let $W$ be a submodule of $V$ maximal subject to $U \cap W=0$. Then $V / W$ is monolithic, with $(U+W) / W$ as its unique minimal submodule, and it is sufficient to show that $V / W=(U+W) / W$. Therefore, we may assume without loss of generality that 
$U$ is the monolith of $V$.

Let $A(H), A(G)$ be the images of $k H$ and $k G$ respectively in end $_{k} V$. We shall show that $A(G)$ is a semisimple artinian ring. It will follow that $U$ is complemented in $V$, and hence that $U=V$. Consider first $A(H)$. Let $a \in A(H)$, and suppose that $U a=0$. Since $V$ is strongly locally completely reducible, there exists a finite subgroup $F$ of $G$ such that $V_{F}$ is completely reducible, $a$ is induced by an element of $k F$, and $G=H F$. We have $V=U \oplus U^{*}$ for some $k F$-module $U^{*}$. Then $V a \leq U^{*}$. Clearly $V a$ is a $k H$-submodule, and it is not hard to deduce that $\sum_{f \in F} V a f$ is a $k G$-submodule of $V$. Since this submodule lies in $U^{*}$, it intersects $U$ trivially, and must be zero. In particular, $V a=0$ and $a=0$.

Therefore $U$ is a faithful $A(H)$-module. But $U_{H}$ is the direct sum of finitely many irreducible $A(H)$-modules, by Clifford's Theorem.

Consequently $A(H)$ is a commutative semisimple artinian ring. Since $A(G)$ is a finitely generated right or left module over its subring $A(H)$, it too is artinian.

Suppose now, if possible, that $I$ is a non-zero nilpotent ideal of $A(G)$. Since $V$ is strongly locally completely reducible, there exists a finite subgroup $F$ of $G$ such that $V_{F}$ is completely reducible and $I \cap A(F) \neq 0$, where $A(F)$ is the image of $k F$ in end $V$. But a nilpotent ideal of $A(F)$ annihilates every completely reducible $A(F)$-module. Hence $I \cap A(F)$ annihilates $V$, and is zero. We have shown that $A(G)$ is semisimple artinian.

(iii) $\Rightarrow$ (i) Suppose that every composition factor of $V$ is complemented, but $\Phi(V) \neq 0$. Let $0 \neq v \in \Phi(V)$. Let $B$ be a submodule of $V$ maximal subject to $v \notin B$, and let $A / B$ be the submodule of $V / B$ generated by $v+B$. Then $A / B$ is the unique minimal submodule of $V / B$, and in particular is a composition factor of $V$. Therefore there exists a submodule $C$ of $V$ such that $V=A+C$ and $A \cap C=B$. Clearly $C$ is a maximal submodule of $V$. Since $v \vDash C$, we have a contradiction. 


\section{An example}

The construction needed for the proof of Theorem $C$ is a special case of another construction given in [7]. The ingredients will consist of a U-group $G$ containing no elements of order $r \quad(r$ being the prime assigned in the statement of the theorem), and an irreducible $\underline{M}_{c}$-module $V$ over $Z_{r} G$ such that $V$ is infinite-dimensional over its endomorphism ring $E$. The latter is of course a division ring, by Schur's Lemma. Such a group and module were in fact constructed in [4], Section 7 . We briefly recall the details of the construction.

First, using Dirichlet's Theorem on the primes in an arithmetic progression, we construct two disjoint infinite sequences $\left\{p_{1}, p_{2}, \ldots\right\}$, $\left\{q_{1}, q_{2}, \ldots\right\}$ of distinct primes, none of which is equal to $r$, such that

$$
q_{1} \cdots q_{i} \mid p_{i}-1
$$

for all $i$.

Let $A_{i}$ be a cyclic group of order $q_{i}^{2}, B_{i}$ be a group of order $p_{i}$, and $A=A_{1} \times A_{2} \times \ldots, B=B_{1} \times B_{2} \times \ldots$. We can represent $A$ by automorphisms of $B$ in such a manner that $\left[A_{i}, B_{j}\right]=1$ if $j<i$, while $\left|A_{i} / C_{A_{i}}\left(B_{j}\right)\right|=q_{i}$ if $j \geq i$. Then the semidirect product $G=B A$ is a U-group, and if $\bar{A}$ denotes the subgroup of $A$ consisting of the elements of square-free order, then $C=B \bar{A}$ is a locally cyclic subgroup of $G$ containing all the elements of prime order of $G$. Now there is a monomorphism of $C$ into the multiplicative group of the algebraic closure $\bar{Z}_{p}$ of $Z_{p}$, and by using this monomorphism we can view the additive group of $\bar{Z}_{r}$ as a $Z_{r} C$-module $X$ on which each non-trivial element of $C$ operates fixed point freely. Let $V$ be any composition factor of $X^{G}$. Since $C \triangleleft G$, every non-identity element of $C$ is fixed point free on $X^{G}$ and hence on $V$. For $C$ is an $r^{\prime}$-group, and so the fixed points of any element of $C$ in a $Z_{r} C$-module are preserved by homomorphisms of the module.

It follows that every element of prime order in $G$ operates fixed 
point freely on $V$, so that $V$ is clearly an $\underline{M}_{c}$-module over $Z_{p} G$, and is furthermore faithful for $G$. Let $E$ be the endomorphism ring of $V$. By the Jacobson Density Theorem, $E$ is a homomorphic image of a subring of $Z_{r} G$. Now every finite set of elements of $Z_{r} G$ generates a finite subring, and hence the same holds for $E$. By Wedderburn's Theorem on finite division rings, $E$ is a field. If $V$ were finite-dimensional over $E$, it would now follow from a modular analogue of Jordan's Theorem on periodic linear groups $([12], 9.4)$, and the fact that $G$ operates faithfully on $V$, that $G$ were almost abelian. This is not the case, and so we now have

LEMMA 3.1. With the above notation, $G$ is a U-group, $V$ is an irreducible $M_{c}$-module over $Z_{r} G, E$ is the endomorphism ring of $V$ and $\operatorname{dim}_{E} V$ is infinite.

Theorem $\mathrm{C}$ now follows immediately from

LEMMA 3.2. Let $k$ be a field of characteristic $r \geq 0$, let $G$ be a countable locally finite $r^{\prime}$-group, and let $V$ be an irreducible $\underline{M}_{C}$-module over $k G$ of infinite dimension over its endomorphism ring. Then there exists an indecomposable $k G$-module $T \geq V$ and an $\underline{\underline{M}}_{c}$-module $W$ over $k G$ such that $T / V$ is irreducible, $\Phi(W)=0$, and $T$ is an epimorphic image of $W$.

Proof. Let $A$ be the natural image of $k G$ in end $V$. Then $A$ is a locally Wedderburn algebra over $k$ in the sense that every finite set of elements of $A$ lies in a semisimple $k$-subalgebra of $A$ of finite dimension over $k$. For every such set of elements lies in the image of the group algebra $k F$ of some finite subgroup $F$ of $G$. The group algebra $k F$ is semisimple by Maschke's Theorem, and so are its homomorphic images. Clearly, $A$ has countable dimension over $k$.

If $0 \neq v \in V$, then the map $a \rightarrow v a(a \in A)$ determines an epimorphism of right $A$-modules from $A$ to $V$ with kernel the annihilator $M(v)$ of $v$ in $A$. Thus $M(v)$ is a maximal right ideal of $A$, and

$$
A / M(v) \cong V
$$

as right $A$-modules. Clearly 


$$
\bigcap_{0 \neq v \in V} M(v)=0
$$

Since $V$ has infinite dimension over its endomorphism ring, Theorem $\mathrm{Cl}$ of [7] shows that there exists an indecomposable $A$-module $T$ containing $V$ such that $T / V$ is irreducible. Clearly $T$ is cyclic, being generated by any element not lying in $V$, and so there exists an epimorphism of right $A$-modules from $A$ to $T$.

Since we have an obvious epimorphism from $k G$ to $A$, we may use that epimorphism to view the $A$-modules above as $k G$-modules. Writing $W$ for the $k G$-module $A,(7)$ and (8) tell us that $\Phi(W)=0$. Also, the $k G$-module $T$ is an epimorphic image of $W$.

It remains to see that $W$ is an $\underline{M}_{c}$-module over $k G$. Let $H$ be a subgroup of $G$. Since $V$ is an $\underline{\underline{M}}_{c}$-module and $G$ has no elements of order $r=\operatorname{char} k, H$ contains a finite subgroup $F$ such that $C_{V}(H)=C_{V}(F)$. Let $Y=C_{W}(F)$. Then, for each $0 \neq v \in V$, $(Y+M(v)) / M(v)$ lies in the centralizer of $F$ in $W / M(v)$. Because of the isomorphism (7), which now becomes $W / M(v) \cong V, H$ operates trivially on $(Y+M(v)) / M(v)$. Thst is, if $y \in Y$ and $h \in H$, then $y(h-1) \in M(v)$. By (8), $y(h-1)=0$. Thus $C_{W}(F) \leq C_{W}(H)$. The reverse inclusion being obvious, it follows that $W$ is an $\underline{\underline{M}}_{C}$-module.

\section{References}

[1] Daniel R. Farkas and Robert L. Snider, "Group algebras whose simple modules are injective", Trans. Amer. Math. Soc. 194 (1974), $241-248$.

[2] A.D. Gardiner, B. Hartley and M.J. Tomkinson, "Saturated formations and Sylow structure in locally finite groups", J. Algebra 17 (1971), 177-211.

[3] J.D. Gillam, "Cover-avoid subgroups in finite solvable groups", $J$. Algebra 29 (1974), 324-329.

[4] B. Hartley, "Sylow subgroups of locally finite groups", Proc. London Math. Soc. (3) 23 (1971), 159-192. 
[5] B. Hartley, "A class of modules over a locally finite group I", J. Austral. Math. Soc. 16 (1973), 431-442.

[6] B. Hartley, "A class of modules over a locally finite group II", $J$. Austral. Math. Soc. Ser. A 19 (1975), $437-469$.

[7] B. Hartley, "Injective modules over group rings", submitted.

[8] L.G. Kovács and M.F. Newman, "Direct complementation in groups with operators", Arch. Math. (Basel) 13 (1962), 427-433.

[9] Donald S. Passman, Infinite group rings (Pure and Applied

Mathematics, 6. Marcel Dekker, New York, 1971).

[10] J.S. Richardson, "Primitive idempotents and the socle in group algebras of periodic abelian groups", submitted.

[11] M.J. Tomkinson, "Prefrattini subgroups and cover-avoidance properties in U-groups", Conad. J. Math. 27 (1975), 837-851.

[12] B.A.F. Wehrfritz, Infinite Zinear groups. An account of the grouptheoretic properties of infinite groups of matrices (Ergebnisse der Mathematik und ihrer Grenzgebiete, 76. Springer-Verlag, Berlin, Heidelberg, New York, 1973).

Mathematics Institute,

University of Warwick,

Coventry,

England;

Department of Mathematics,

University of Wisconsin,

Madison,

Wisconsin,

USA. 